Wide-field fluorescence lifetime imaging with optical sectioning and spectral resolution applied to biological samples

\author{
D. S. Elson, J. Siegel, S. E. D. Webb, S. Lévêque-Fort, D. Parsons- \\ Karavassilis, M.J. Cole, and P.M.W. French \\ Femtosecond Optics Group, Department of Physics, Imperial College of \\ Science, Technology and Medicine, Prince Consort Road, London SW7 \\ 2BW, U.K
}

\title{
D. M. Davis
}

Department of Biological Sciences, Imperial College of Science, Technology and Medicine, London SW7 2BW, U.K

\section{J. Lever}

Department of Bioengineering, Imperial College of Science, Technology and Medicine, Prince Consort Road, London SW7 2BY, U.K

\author{
R. Juškaitis, M. A. A. Neil, L. O. Sucharov and T. Wilson \\ Department of Engineering Science, University of Oxford, Parks Road, \\ Oxford OX1 3PJ, U.K.
}

Contact: Daniel Elson, ds.elson@ic.ac.uk.

Tel. $+44(0) 2075947745$

Fax $+44(0) 2075947782$ 


\section{Abstract}

We have performed wide-field fluorescence lifetime imaging with spectral resolution and optical sectioning to achieve five-dimensional fluorescence microscopy. Spectral filtering has been shown to have the potential to provide functional information about biological tissue by simultaneously measuring the spectral/lifetime signature of the sample. The potential to use multispectral imaging to spatially separate cellular components by their different emission wavelengths has also been demonstrated thus reducing artifacts in the calculated lifetime maps. The instrument is based on diodepumped solid-state laser technology and an ultrafast gated optical image intensifier. We also report the use of a picosecond blue laser diode as the excitation source to produce a fluorescence lifetime microscope with a footprint of less than $1 / 4 \mathrm{~m}^{2}$. 


\section{Introduction}

The characteristic decay rate of the fluorescence emitted by a molecule after excitation with a laser pulse is dependent on the type of fluorophore present and its environment. This so-called fluorescence lifetime can be expressed in terms of the radiative and non-radiative decay rates and, since the non-radiative decay rate depends upon the local environment that the fluorophore is in, a fluorescence lifetime measurement can reveal and quantify the presence of certain analytes, eg $[\mathrm{Ca} 2+]^{1}$, $[\mathrm{PO} 2+]^{2}, \mathrm{pH}^{3}$. Fluorescence lifetime imaging (FLIM) simultaneously measures the lifetime of every point in a two-dimensional image and can reveal complementary information to standard fluorescence intensity microscopy.

It is also desirable to be able to record spectroscopic information about the sample in order to improve lifetime contrast or fluorophore specificity. In order to image the fluorescence lifetime whilst resolving its spectral content we have developed a relatively low-cost, wide-field instrument comprising a gated optical intensifier (GOI), a multi-spectral imager (MI), a CCD camera and an ultrafast home-built laser system ${ }^{4}$.

High resolution optical sectioning can be achieved by the use of a structured illumination technique ${ }^{5}$, which preserves the wide-field nature of the FLIM instrument and brings a number of advantages, such as improved lifetime fitting and improved transverse spatial resolution ${ }^{46}$.

To demonstrate this functionality, we have applied this technique to a test sample made up of different types of fluorescent microspheres. To provide a biological example we have also used the MI to explore the spectral/lifetime characteristics of a piece of rat heart. We have also demonstrated the potential to image and spatially separate the different components of a cell using spectral discrimination. Finally we 
include preliminary results obtained by using a picosecond blue laser as the illumination source in our wide-field FLIM instrument.

\section{Experimental set-up}

\subsection{FLIM instrument}

The temporal resolution of the FLIM instrument is provided by using a GOI (Kentech Instruments, UK) in combination with a home-built ultrafast oscillator/amplifier system. The GOI consists of a photocathode that converts incident photons into electrons, which are then amplified by a microchannel-plate and are incident upon a phosphor screen. The resulting intensity distribution is imaged onto an 8-bit intensified CCD camera (Photonic Science, UK). A high voltage spike is applied to the wire mesh in front of the photocathode so that the GOI essentially acts like a time gate with a temporal FWHM of 90 ps. By recording a series of fluorescence intensity images from a sample at different delay times and computationally fitting a single exponential decay to each pixel, a fluorescence lifetime map can be generated.

\section{[Insert figure 1 about here]}

The laser source used is a home-built diode-pumped Cr:LiSAF oscillator/amplifier system which is described in more detail elsewhere ${ }^{7}$ Briefly, the oscillator consists of a $5 \mathrm{~mm}$ Brewster-angled rod in a z-fold cavity pumped by two 500 $\mathrm{mW}, 670 \mathrm{~nm}, 100 \mu \mathrm{m}$ stripe width laser diodes. The resulting output of $80 \mathrm{MHz}, 100 \mathrm{fs}$ pulses at $860 \mathrm{~nm}$ is fed through an isolating seed path onto a thin film polarizer, injecting the pulses into the amplifier cavity. A Pockel's cell then selectively changes 
the polarization state of the pulses such that one pulse is selectively amplified by the gain medium, consisting of a $6 \mathrm{~mm}$ plane/Brewster-angled rod pumped by two of the $500 \mathrm{~mW}$ diodes described above and one $350 \mathrm{~mW}, 50 \mu \mathrm{m}$ stripe width diode. This amplified pulse is switched out of the cavity, and typically has a pulse energy of $1.5 \mu \mathrm{J}$ and a duration of $10 \mathrm{ps}$. The repetition rate of the amplifier can be set up to $20 \mathrm{kHz}$ and this determines the maximum possible resolvable lifetime of the FLIM instrument. Although self-phase modulation in the Pockel's cell and gain medium increases the length of the pulse, recompression is not necessary because the pulse length is still significantly shorter than the gatewidth of the GOI. After amplification the pulse is frequency doubled to $430 \mathrm{~nm}$ using a BBO crystal, and then directed into the microscope to excite the specimen.

\section{$2.2 \quad$ Optical sectioning}

In order to obtain thin optical sections of the specimen we have implemented a wide-field technique using structured illumination. In a confocal microscope system, only in-focus light is imaged because the optical transfer function (OTF) attenuates the collected light rapidly with defocus. In a conventional wide-field microscope it is only the zero spatial frequency component of the collected light that is not attenuated with defocus - but typically this d.c. background blurs the image, and degrades the sectioning. By imaging an alternately transparent and opaque bar grating directly into the sample, it is possible to produce a spatially modulated excitation and a resulting fluorescence distribution. Sectioned images can be obtained in a regular wide-field microscope by extracting only the spatially modulated information from the recorded image, $I_{1}$. In order to remove the grating intensity pattern from this image, it is 
necessary to record two further images $\left(\mathrm{I}_{2}\right.$ and $\left.\mathrm{I}_{3}\right)$, in which the grating has been translated laterally by spatial frequencies of $120^{\circ}$ and $240^{\circ}$ relative to its original position.

It is then possible to recreate the optical section by calculating ${ }^{5}$

$$
\mathrm{I}_{\mathrm{s}}=\left(\frac{3}{\sqrt{2}}\right)\left[\left(\mathrm{I}_{1}-\mathrm{I}_{2}\right)^{2}+\left(\mathrm{I}_{1}-\mathrm{I}_{3}\right)^{2}+\left(\mathrm{I}_{2}-\mathrm{I}_{3}\right)^{2}\right]^{1 / 2}
$$

and the conventional image can be calculated using

$$
\mathrm{I}_{0}=\mathrm{I}_{1}+\mathrm{I}_{2}+\mathrm{I}_{3}
$$

A sectioned FLIM map can be produced if three images are recorded and the above algorithm used at each discrete time gate ${ }^{8}$.

In practice, a square grating consisting of alternately transparent and opaque strips is used, because this is more light efficient than a sinusoidally varying one. Its transmission can be expressed as

$$
\mathrm{T}=\frac{1}{2}+\frac{2}{\pi}\left(\cos (2 \pi v x+\phi)-\frac{1}{3} \cos (3(2 \pi v x+\phi))+\frac{1}{5} \cos (5(2 \pi v x+\phi))-\ldots\right)
$$

where $v$ is the grating period and $\varphi$ is an arbitrary phase. The spatial frequency of the grating can be chosen in such a way that the fifth and higher harmonics make a negligible contribution to the image, because the OTF tails off at higher spatial frequencies. There may still be a significant contribution from the third harmonic component, but (1) will filter this from the sectioned image. The limitations of this technique are mainly related to the larger acquisition times and lower light efficiency compared with conventional (unsectioned) FLIM, leading to an increased chance of laser illumination fluctuations, or lateral movements of the sample and grating. It is important to use a grating with an equal mark space ratio, otherwise the second and fourth harmonic components can lead to errors in the sectioning algorithm. 


\subsection{Multispectral imager}

In order to gain more functional information from a biological sample, it is desirable to be able to resolve different spectral components of the fluorescence, as well as its lifetime 9 . To this end, we have incorporated a commercially available MI into the fluorescence microscope. This device splits the field of view into two spectrally discrete bands by means of a dichroic beam splitter, as shown in figure 1, and then images the two bands side-by-side onto a detector - in our case this is the GOI. The MI is mechanically robust and does not significantly increase the complexity of the system, particularly because only one wide-field detector is required. Filters can be placed after the dichroic beam splitter to reduce the pass bands further if required, as well as to adjust for the different intensity levels of the two bands.

\section{Results}

\subsection{5-D fluorescence of microspheres}

We have used the spectrally-resolved 3-D FLIM microscope to analyse a sample made up of $4.5 \mu \mathrm{m}$ and $15 \mu \mathrm{m}$ fluorescent microspheres (Molecular Probes Europe, The Netherlands), which emit in the green and the blue spectral regions respectively. The microspheres are held in immersion oil between a glass slide and a cover slip, and are imaged with a $x 60, \mathrm{NA}=0.8$ objective lens. The conventional (non-sectioned) image is shown in figure 2(a), where the left and right hand sides of each image show the same field of view, but represent a different spectral pass band, in this case (450-505nm) and (505-800nm) respectively. Therefore the green fluorescent microspheres appear on the right hand side and the blue fluorescent microspheres on the left, although the blue ones also appear weakly on the right because their emission spectrum extends into the green. 
[Insert figure 2 about here]

The conventional FLIM map (figure 2(b)) shows that the small microspheres in the right hand sub-image have a longer lifetime than the large ones, which appear on the left. However, since the large microspheres also appear weakly in the right hand subimage, there is a transition in lifetimes at the boundary between the two types of microsphere, despite the fact that only two different lifetimes are expected.

The sectioned intentity image is shown in figure 2(c), which shows improved out-of-focus light rejection compared to the conventional image. It can clearly be seen on the green filter channel of this image that there are three rogue beads of intermediate size $(\sim 10 \mu \mathrm{m})$. In fact, in the sectioned fluorescence lifetime map (figure $2(\mathrm{~d}))$ it can be seen that these rogue beads have the same lifetime as the small beads, as well as emitting in the same wavelength band, indicating that they have the same fluorescent coating. It can be seen that the sectioning has a greater visible effect on the spatial resolution of the FLIM map than the intensity image, mainly because FLIM does not preserve the intensity contrast between pixels, as long as they are above a certain value.

\subsection{FLIM of biological tissue}

In order to investigate the application of multispectral FLIM to biological tissue we have measured the autofluorescence lifetime of a freshly extracted piece of rat heart. By placing different filters into the MI, different spectral pass-bands could be imaged independently, giving a characteristic spectral/lifetime signature that will be more tissue-specific than spectral or lifetime information alone. The rat heart was imaged 
using a X10 microscope objective and an intensity image is shown in figure 3(a). The specimen was chosen to have very little structure evident at this magnification as is indicated by the homogeneity of the lifetime maps shown with different spectral passbands in figures 3(b)-(d). However, it is clear that there is a net change in lifetime between each spectrally resolved FLIM map, which can be quantified by averaging the lifetimes of a region of each image. For example, it is clear that the average lifetime of the green spectral pass-band (right hand sub-image of figure $3(\mathrm{c})$ ) is much longer than that of the red (right hand sub-image of figure 3(d)). After averaging a 50x50 pixel area the following lifetimes were obtained: $>685 \mathrm{~nm}, 495 \pm 33 \mathrm{ps} ; 505-675 \mathrm{~nm}, 784 \pm 55$ ps; $>505 \mathrm{~nm}, 636 \pm 70 \mathrm{ps} ;<505 \mathrm{~nm}, 583 \pm 41 \mathrm{~nm}$. The MI can thus be used to increase the information recorded from heterogeneous tissue by measuring the tissue-specific lifetime for different wavelength bands.

[Insert figure 3 about here]

\subsection{FLIM of cells}

By tagging different components of cells with different fluorescent dyes, we can potentially use both lifetime and multi-spectral imaging to contrast different cellular regions. As a preliminary demonstration, we used $721.211 \mathrm{~B}$ cells that have been transfected with a specific MHC protein (HLA-B8). The cells were fixed with paraformaldehyde, which kills the cells and covalently bonds the molecules together. Detergent was then added to make the cell membranes permeable. After the cells had been washed, various fluorescent markers may be introduced. For instance, the blue DNA dye may be used to label the DNA in the nucleus, and the green Alexa 430 can be 
covalently conjugated to a second antibody that specifically binds to the first antibody labeling the MHC protein.

\author{
[Insert figure 4 about here] \\ [Insert figure 5 about here]
}

Figures 5(a) and 5(b) show the conventional image and FLIM map of a cell whose membrane has been stained with the Alexa 430 dye, and so it appears in the right hand sub-image (green channel). A weak autofluorescence signal from the cell nuclei can be seen in figure 5(b), which is indicated by the white arrows. This mainly occurs in the left hand sub-image (blue channel) and shows an important application of the MI: without this spectral 'rejection' of the autofluorescence of the nuclei, the lifetime of the desired fluorescence from the tagged membrane would be calculated incorrectly. This is illustrated in figure 5(c) where the two spectral pass-bands have been combined computationally and the resultant lifetime map calculated. It can be seen that the two regions marked by arrows, corresponding to the positions of the nuclei, have a different (longer) lifetime than the surrounding cell, an effect which is not present on the right hand sub-image of figure 5(b).

Figures 5(d) and 5(e) show FLIM maps of cells whose nuclei have been stained with the blue Sotox DNA dye. We note from figure 4 that the emission spectrum extends into both spectral pass-bands of the MI. Hence the nuclei appear in both spectral sub-images of the MI. It should be observed that, importantly, the fitted lifetimes are the same for both pass-bands. This indicates the potential to obtain useful lifetime information from appropriately spectrally resolved images that select different 
fluorophore labels, e.g. to separate the nucleus from the rest of the cell..

\subsection{FLIM using a blue ps diode}

Recent work has focused on the miniaturisation of the FLIM instrument ready for clinical deployment together with coupling to an endoscope. The diode-pumped oscillator/amplifier system has a relatively large footprint, but by increasing the repetition rate of the detection system the output from an oscillator or pulsed laser diode can be used. Pulsed laser diodes have been used mainly to perform single-point fluorescence lifetime measurements using time-correlated single photon counting techniques. The limited application of (only red and infrared) pulsed laser diodes to fluorescence lifetime imaging has been in a pixel-by-pixel confocal scanning mode and either no lifetime images have been shown or these showed $\operatorname{artifacts}^{10}{ }^{11}$. We report here preliminary wide-field FLIM with a blue laser diode.

A High Rate Imager (HRI, Kentech Instruments Ltd., UK), which operates at up to $110 \mathrm{MHz}$, with a $200 \mathrm{ps}$ minimum gate width $^{12}$, was used in place of the GOI. The blue pulsed laser diode (400nm, <30 ps, $40 \mathrm{MHz}, 1 \mathrm{~mW}$ average power) available commercially from PicoQuant $\mathrm{GmbH}$, reduces the total footprint of the microscope system to $<1 / 4 \mathrm{~m}^{2}$. The diode laser output was coupled into a fibre and was used to illuminate sections of unstained rat ear in a microscope.

Figures 6(a) and 6(b) show a X20 objective intensity image and FLIM map of a $15 \mu \mathrm{m}$ thick section of rat ear. The honeycomb-like structure seen on the left hand side of the image is elastic cartilage, which can be seen to have the longest fluorescence lifetime. There is an elongated blood vessel cross-section near the top right corner, which has a short lifetime, and it can be seen that its inner and outer walls have a longer 
lifetime. Figures 6(c) and 6(d) show the top of the vessel in more detail and are taken with a X100 objective lens. The congealed blood in the vessel (short lifetime) contrasts strongly with the inner and outer vessel walls (long lifetimes). In our ongoing work we have also used the blue laser diode to macroscopically image wellplate assays of chemically and biologically interesting fluorophores, the results of which will be presented elsewhere. This will have important applications in high-throughput drug screening or DNA sequencing. ${ }^{13}$

[Insert figure 6 about here]

\section{Conclusion}

We have demonstrated several applications of a spectrally- and depth-resolved wide-field fluorescence lifetime microscope. The instrument has been made considerably more compact and more widely deployable by replacing the usual diodepumped solid-state laser system/Ti:Sapphire oscillator with a blue picosecond pulsed laser diode, producing a FLIM microscope system of $<1 / 4 \mathrm{~m}^{2}$ footprint. The importance of rejection of out-of-focus light to FLIM has been demonstrated using a fluorescent microsphere sample. The multispectral imager has been applied to spatially separate the components of cells and to probe the spectral/lifetime signature of a piece of rat heart.

\section{Acknowledgments}

The authors acknowledge PicoQuant GmbH for the loan of the blue pulsed laser 
diode. Funding for this research was provided by the UK Engineering and Physical Sciences Research Council (EPSRC) and the Biotechnology and Biological Sciences Research Council (BBSRC). D. S. E. and M. J. C. acknowledge an EPSRC CASE studentship supported by Kentech Instruments Ltd. and Royal Marsden Hospital Trust respectively. S. E. D. W. and D. P-K. acknowledge an EPSRC studentship.

\section{References}

${ }^{1}$ Lakowicz, J. R., Szmacinski, H., and Johnson, M. L., 1992, J. Fluorescence, 2, 47

${ }^{2}$ Bambot, S. B., Rao, G., Romauld, M. M., Carter, G. M., Sipior, J., Terpetching, E., and Lakowicz, J. R., 1995, Biosens. Bioelect., 10, 643

${ }^{3}$ Sanders, R., Draaijer, A., Gerritsen, H. C., Houpt, P. M., and Levine, Y. K., 1995, Anal. Biochem., 227, 302

${ }^{4}$ Siegel, J., Elson, D. S., Webb, S. E. D., Parsons-Karavassilis, D., Lévêque-Fort, S., Cole, M. J., Lever, Neil, M. A. A., Juškaitis, R., Sucharov, L. O., Wilson, T., and French, P. M. W., 2001, Opt. Lett, 26, 1338

${ }^{5}$ Neil, M. A. A., Juškaitis, R., and Wilson, T., 1997, Opt. Lett., 22, 1905

${ }^{6}$ Squier, A., and Bastiaens, P. I. H., 1999, J. Microsc., 193, 36

${ }^{7}$ Parsons-Karavassilis, D., Jones, R., Cole, M. J., French, P. M. W., and Taylor, J. R., 2000, Opt. Comm., 175, 389

${ }^{8}$ Cole, M. J., Siegel, J., Webb, S. E. D., Jones, R., Dowling, K., French, P. M. W., Lever, M. J., Sucharov, L. O. D., Neil, M. A. A., Juškaitis, R., and Wilson, T., 2000, Opt. Lett., 25, 1361

${ }^{9}$ Hewett, J., McKechnie, T., Sibbett, W., Ferguson, J., Clark, C., and Padgett M., 2000, J. Mod. Opt., 47, 2021 
${ }^{10}$ Waddell, E., Wang, Y., Stryjewsky, W., McWhorter, S., Henry, A. C., Evans, D.,

McCarley, R. L., and Soper, A., 2000, Anal. Chem., 72, 5907

${ }^{11}$ Lassiter, S., Stryjewsky, W., Legendre, B., L., Erdmann, R., Wahl, M., Wurm, J.,

Peterson, R., Middendorf, L., and Soper, S. A., 2000, Anal. Chem., 72, 5373

${ }^{12}$ Webb, S. E. D., Gu, Y., Lévêque-Fort, S., Siegel, J., Cole, M. J., Dowling, K., Jones,

R., Lever, M., J., Neil, M. A. A., Juškaitis, R., Sucharov, L. O., Wilson, T., and French,

P. M. W., Submitted for publication, Rev. Sci. Inst.

${ }^{13}$ Rogers, M. V., 1997, Drug Discovery Today, 2, 156

Figure captions

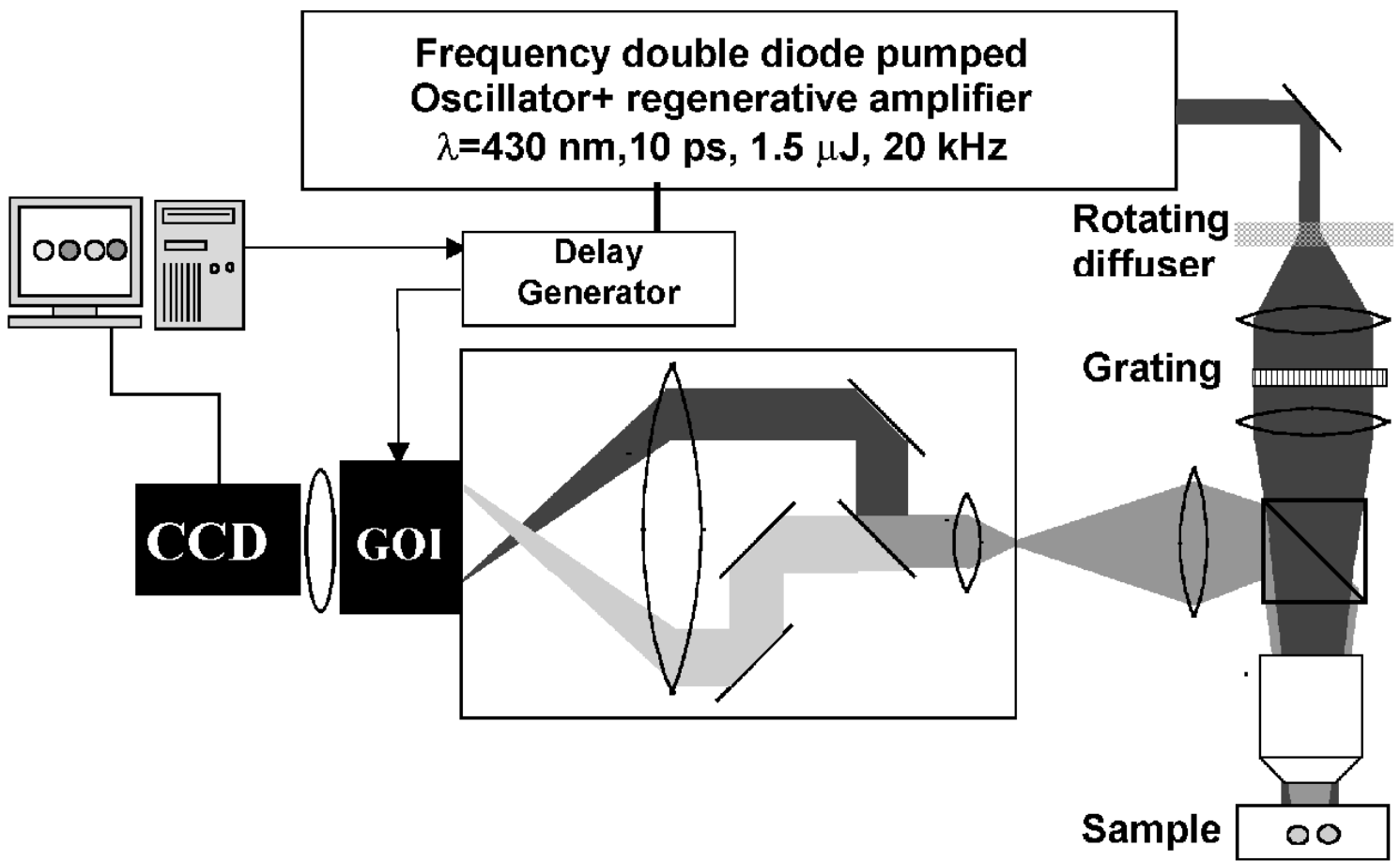

Figure 1. Experimental set-up showing how the MI splits the light spectrally and forms two sub-images side-by-side on the GOI. 
Intensity image
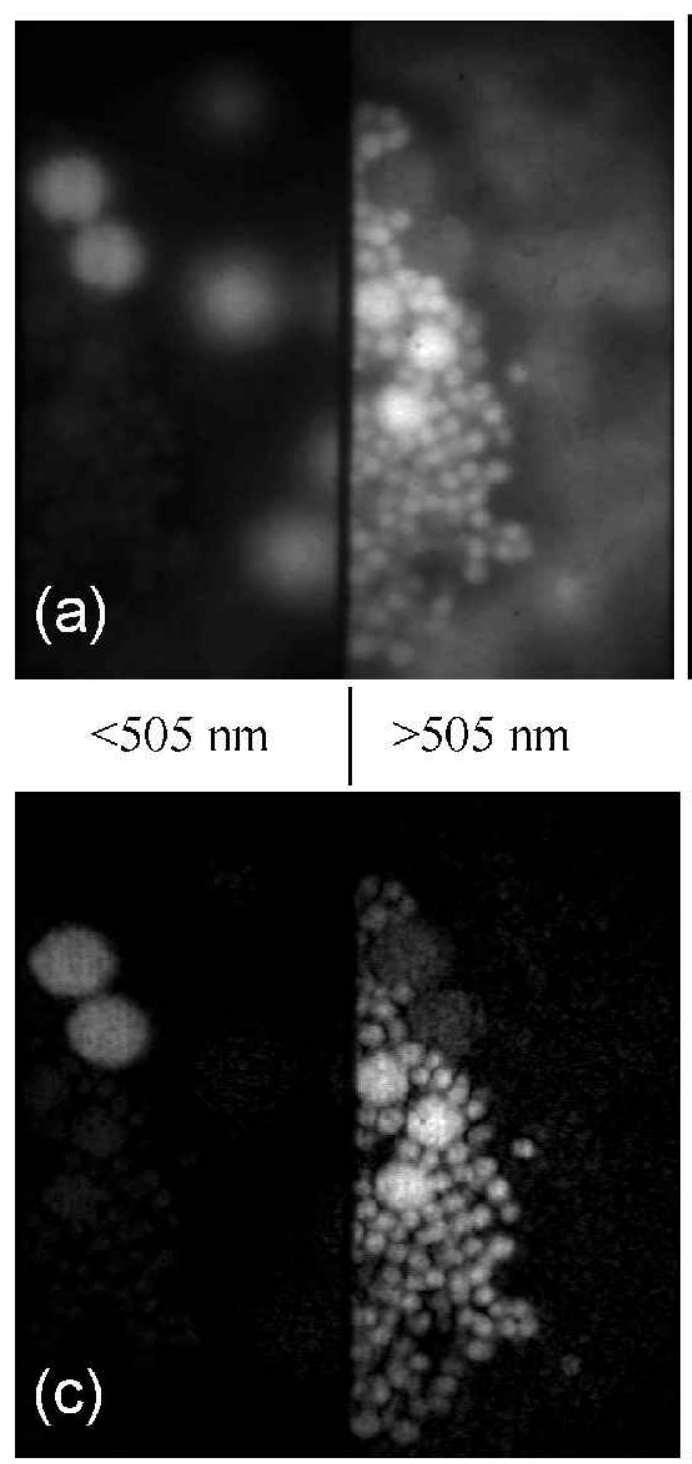

Lifetime map
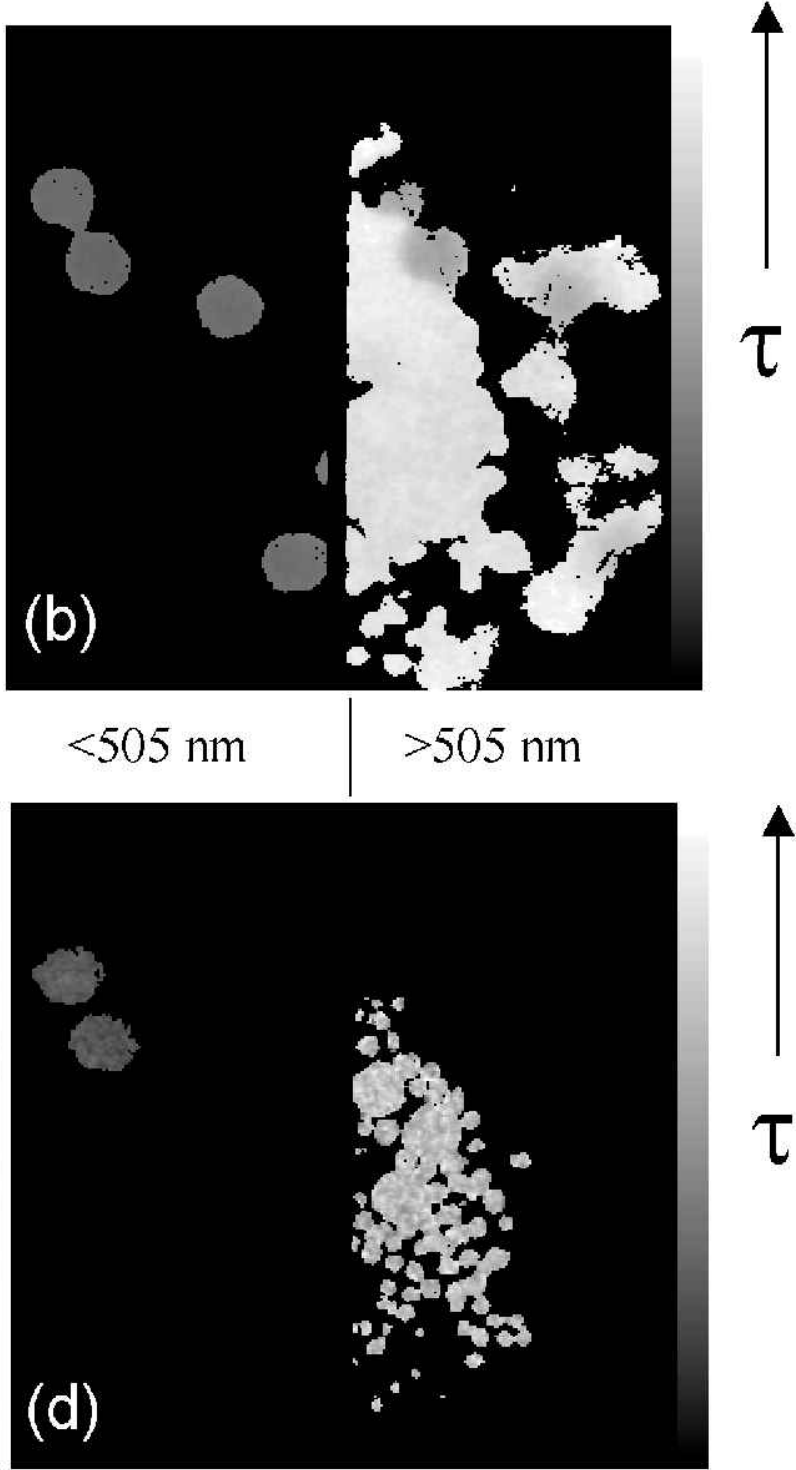

$\tau$

Figure 2. (a) and (b) conventional (unsectioned) intensity and FLIM images of a sample made up of 4.5 and $15 \mu \mathrm{m}$ fluorescent microspheres. (c) and (d) show the same lateral region but the structured illumination method has been used to obtain a sectioned intensity and FLIM image. The lifetime scale is from 1500-5700 ps. 
Intensity image
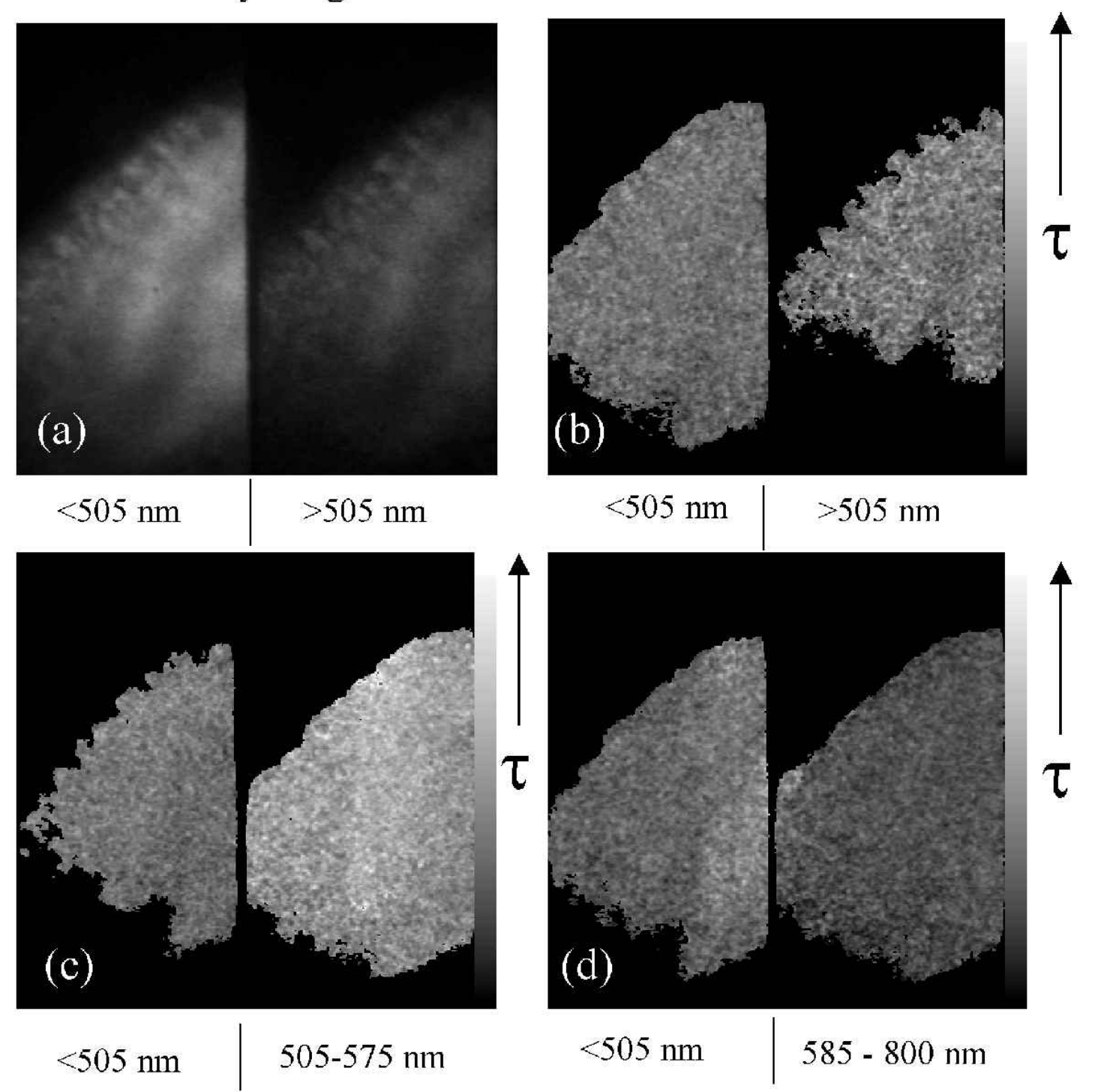

Figure 3. (a) shows an intensity image of a piece of rat heart using the MI to spatially separate the two spectral pass-bands indicated. The FLIM maps are shown in figures (b)-(d) for the different spectral regions indicated and the lifetime scale is $300-1100 \mathrm{ps}$ for each. 


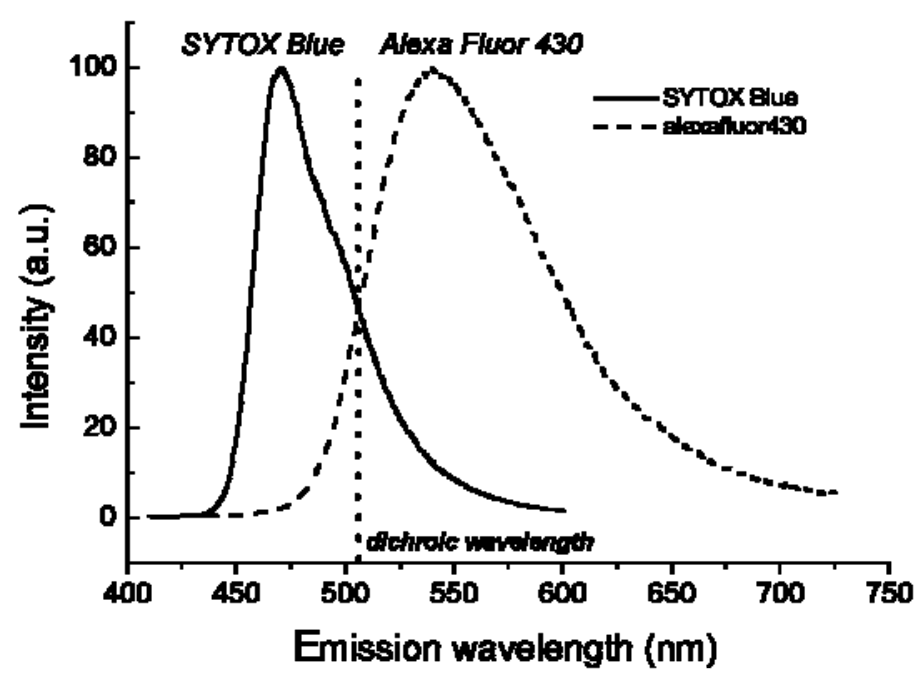

Figure 4. Emission spectra for SYTOX Blue and Alexa Fluor 430 showing significant overlap. The wavelength of the dichroic is indicated and splits the region of emission overlap. 
Intensity image
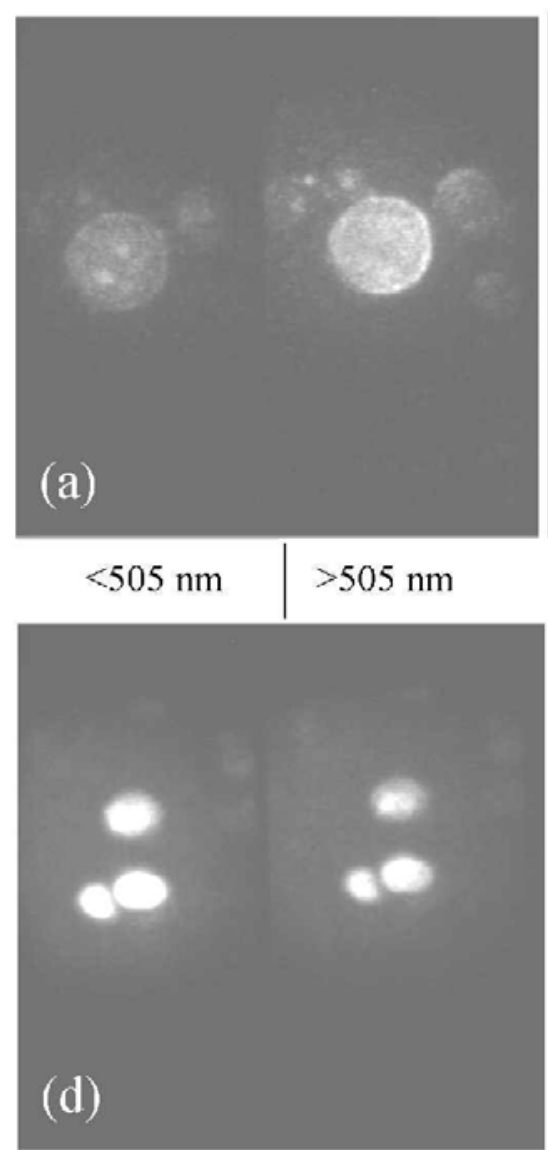

Lifetime map
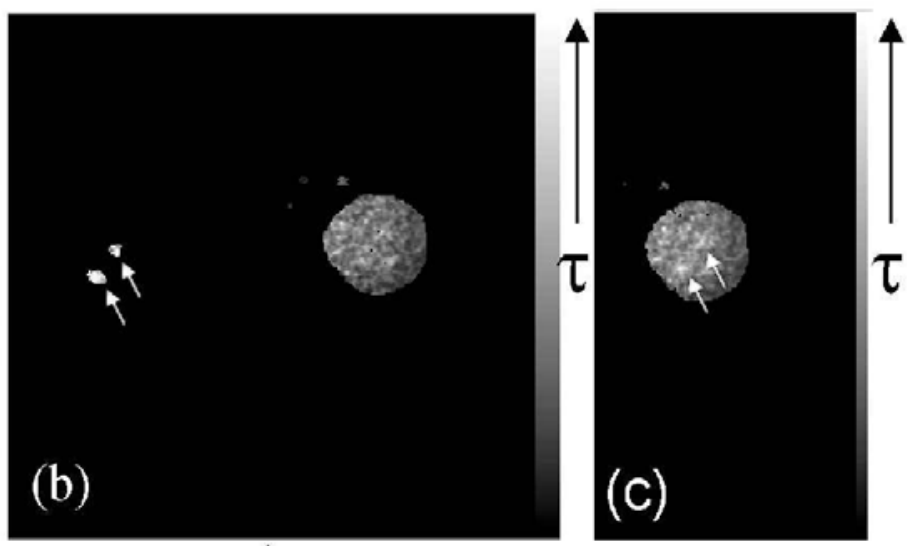

$<505 \mathrm{~nm}$

$>505 \mathrm{~nm}$

$>435 \mathrm{~nm}$

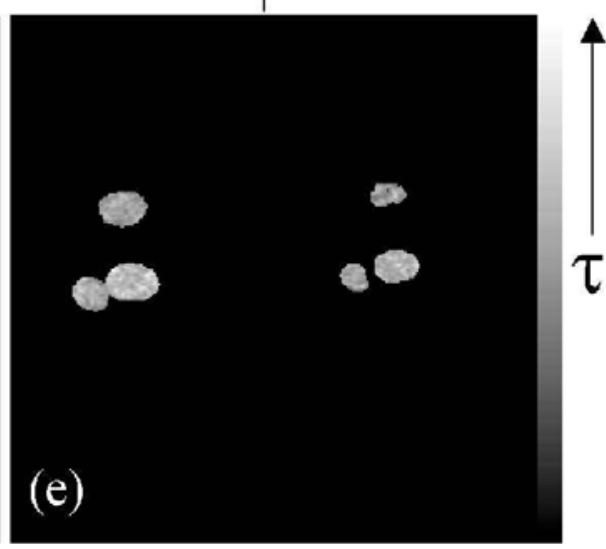

Figure 5. Images of cells stained with ((a)-(c)) Alexa Fluor 430 and ((d) and (e)) SYTOX Blue. The Alexa Fluor 430 stained the cell membranes and emits in the green (right hand sub-image of (b)). The autofluorescence of the cell nuclei, which emit in the blue channel, is seen in the left hand sub-image of (b). In (c), the intensity images of the left hand and right hand sub-images of (b) were combined and a new lifetime map created. The effect of the autofluorescence is to distort the fitted lifetimes (where the regions of interest are indicated by arrows). The SYTOX Blue stained the cell nuclei, which can be seen in figures (d) and (e), but the emission is seen in both pass-bands because the beam-splitter is not optimised. However, the lifetime calculated is the same for the two different pass-bands. The lifetime scale is 500-5000 ps. 


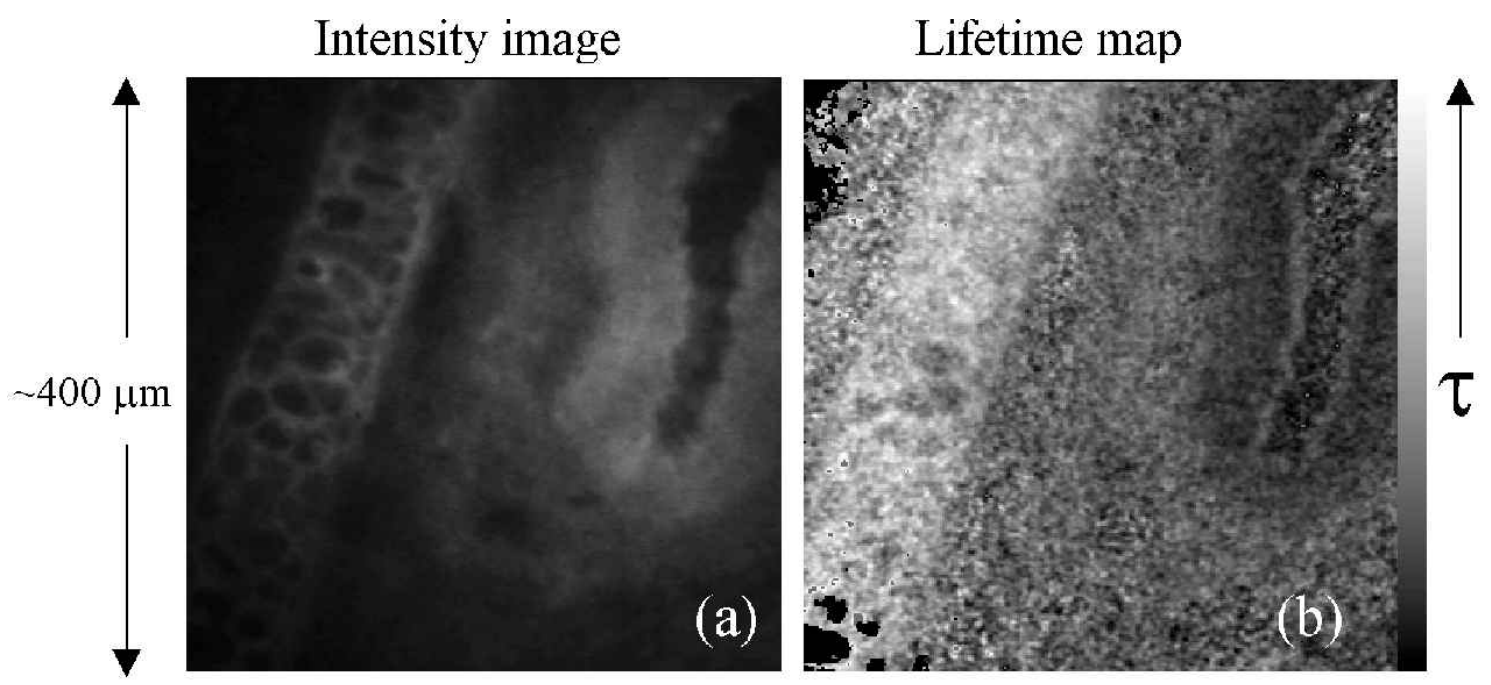

Figure 6. Intensity and lifetime maps of autofluorescence from a $15 \mu \mathrm{m}$ section of rat ear with (a) and (b) X20 objective, and (c) and (d) X100 objective. The lifetime scale is $1150-3500 \mathrm{ps}$ in (b) and 1100-2300 ps in (d). 